\title{
Engagement, satisfacción académica y valores morales en estudiantes universitarios
}

\section{Engagement, academic satisfaction and moral values in university students}

Jaquelinne Reséndiz Chávez

Marco Antonio De La Cruz Pérez

Angélica Romero Palencia

Universidad Autónoma del Estado de Hidalgo (UAEH)

\section{Resumen}

En años recientes, la educación superior en México ha tenido mayor su demanda y, al ser un elemento importante en el desarrollo de nuestra sociedad, se ha generado el interés por estudiar los elementos y actores que en ella convergen. En dicho contexto, el objetivo de este estudio fue correlacionar las variables de engagement, valores morales y satisfacción académica en estudiantes universitarios de psicología de la Universidad Autónoma del Estado de Hidalgo (UAEH). La muestra fue de 215 estudiantes del Instituto de Ciencias de la Salud, a quienes se les aplicó el Utrecht Work Engagement Scale México (UWES-S México, por sus siglas en inglés), la Escala de Satisfacción Académica y el Cuestionario de Valores Morales en la Formación y el Ejercicio Profesional. La prueba estadística, producto-momento de Pearson, mostró la existencia de correlaciones estadísticamente significativas entre las tres variables, sin embargo, éstas no estuvieron presentes en todos los factores. Además, las correlaciones fueron bajas, lo cual significa que la relación existente entre las variables es poca, en sí se encuentra presente. Desde la psicología estos hallazgos aportan nuevos elementos a la literatura de valores morales y satisfacción académica, y permiten una mejor comprensión del engagement al integrar nuevas variables en su estudio.

Palabras clave: engagement, valores morales, satisfacción académica, psicología positiva, universitarios.

Nota del autor

Jaquelinne Reséndiz Chávez, Facultad de Psicología, Universidad Autónoma del Estado de Hidalgo (UAEH).

La correspondencia en relación con este artículo debe dirigirse a Jaquelinne Reséndiz Chávez, Facultad de Psicología, UAEH, edificio Torres de rectoría, Carretera Pachuca-Actopan, km. 4.5 7o. piso, colonia Campo de Tiro, C.P. 42039, Pachuca de Soto, Hidalgo, México.

Dirección electrónica: noeliabeatriz9@hotmail.com 


\begin{abstract}
In recent years, higher education in Mexico has increased its demand and being an important element in the development of our society, has generated the interest to study the elements and actors that converge in it, reason why, the aim of this Study was to correlate the variables of engagement, moral values and academic satisfaction in university students of psychology at the Universidad Autónoma del Estado de Hidalgo. The sample was 215 students of the Institute of Health Sciences, to whom they were applied, the Utrecht Work Engagement Scale-Mexico (UWES-S Mexico), the Scale of Academic Satisfaction and the Moral Values Questionnaire in Training and Exercise Professional. The statistical test, Pearson product-moment, showed the existence of statistically significant correlations between the three variables, however, were not present in all factors. In addition, the correlations were low, which means that the influence between the variables is small, but it is definitely present. These findings bring new elements, from psychology, to the existing literature of moral values and academic satisfaction, and allow a better understanding of engagement by integrating new variables into their study.

Keywords: engagement, moral values, academic satisfaction, positive psychology, university students.
\end{abstract}

Hace más de medio siglo, la calidad de las instituciones universitarias se daba por sentada y no era sujeta a evaluación por el Estado ni por la sociedad (Aguila, 2005). El interés por evaluar las universidades, en América Latina, surge en los años 90, debido a cuestiones como la restricción del financiamiento estatal a las universidades, cuestionamientos sobre los recursos invertidos y resultados obtenidos en relación con la cantidad de graduados, el aumento de la demanda educativa y el surgimiento de instituciones privadas de educación superior (Hernández, Lara, Ortega, Martínez, \& Avelino, 2010).

Con respecto a la demanda educativa, datos del Instituto Mexicano para la Competitividad (IMCO, 2016), señalan que un total de 335,110 personas han estudiado psicología en México, lo que coloca a la carrera en el séptimo puesto de profesiones con mayor demanda. En el estado de Hidalgo, la matrícula total de la carrera de psicología es de 3,334, de los cuales egresan 501 , esto coloca a la profesión en el tercer lugar de matriculados y cuarto lugar de egresados del total de 16 carreras que se ofertan en el estado (Observatorio Laboral, 2014).

Debido a estas demandas y retos, las universidades se han visto en la necesidad de mostrar su calidad mediante la acreditación (Garbanzo, 2007). A nivel mundial se encuentran organismos como la Organización para la Cooperación y el Desarrollo Económicos (OCDE), mientras que, en México, se encuentra el Consejo para la Acreditación de Programas Académicos [COPAES] (Hernández et al., 2010) y el Sistema Nacional de Evaluación, 
Acreditación y Certificación (SINEACE), apoyado por organismos evaluadores como el Centro Nacional de Evaluación para la Educación Superior (CENEVAL), entre otros (Álvarez, Chaparro, \& Reyes, 2014).

Sin embargo, los indicadores de evaluación de estos organismos se centran en los conocimientos académicos (OCDE, 2015), plan de estudios, infraestructura, equipamiento, gestión administrativa y financiera (Universidad de Guadalajara, s. f.), cantidad de profesores, horas de clase, grados académicos de los profesores, cantidad de investigaciones, oferta académica, entre otros (El Economista, 2016). Esto deja de lado variables tales como el bienestar del alumno, su satisfacción con la institución educativa y los valores morales en su formación académica.

Con base en lo anterior, la importancia del estudio de engagement en universitarios radica en que diversos estudios han demostrado su relación con altos niveles de autoeficacia, esperanzayoptimismoen estudiantes(Ouweneel, Le Blanc, \& Schaufeli, 2011), un desempeño escolar superior (Salanova, Schaufeli, Martínez, \& Bresó, 2009), menores niveles de estrés (Casuso, 2011) y menor sensación de malestar físico y psicológico (Salanova, Llorens, Cifré, \& Martínez, 2012). Por otra parte, el estudio de los valores morales, en el contexto de la educación mexicana, ha sido un tema poco abordado (Barba, 2007), siendo que una educación de calidad implica, no sólo adquirir conocimientos, sino ejercer estos conocimientos de manera responsable para brindar un bien a la sociedad (Benois, 2011). Finalmente, al considerar que los valores y ética profesional, aunados a la satisfacción académica, son factores que influyen de manera decisiva en el ejercicio de la profesión, y tomado en cuenta que existe la necesidad de formar alumnos de manera integral con base en su desarrollo personal (Lindsay, 2009), se consideró para este estudio, la variable satisfacción, ya que un alumno satisfecho es el reflejo de la calidad en su formación (Jiménez, Terríquez, \& Robles, 2011). A continuación, se revisan cada una de estas variables.

En primer lugar, el concepto engagement surge de la psicología positiva, cuyo enfoque se centra en el estudio de los aspectos que proporcionan bienestar psicológico y deja de lado los aspectos patológicos de las personas (Vallerand, \& Verner-Filion, 2013). Esta palabra proviene del idioma inglés, que ordinariamente se utiliza como sustantivo (engagement) y adjetivo (engaged); hace referencia al compromiso voluntario, deber o promesa entre dos personas. Como verbo, se refiere a la acción de utilizar o contratar a alguien, atrayendo, persuadiendo u obligando su atención (Sheppard, 2011). En este sentido, la traducción al español de esta palabra es compromiso (Cambridge University Press, 2017), teniendo que, de acuerdo con el diccionario de La Real Academia Española, el compromiso se define como una obligación contraída, palabra dada, dificultad, empeño, o una solución dada por obligación o necesidad para complacer (RAE, 2014). Sin embargo, 
esta definición en español es insuficiente $\mathrm{y}$ está descontextualizada, por lo que se utiliza la palabra en su idioma original, para no alejarla de la idiosincrasia del concepto utilizado en psicología.

En psicología, el término engagement se define como "un estado mental positivo relacionado con el trabajo y se caracteriza por vigor, dedicación y absorción" (Schaufeli, Salanova, González-Roma, \& Bakker, 2002, p. 74). Además de ser un estado cognitivo envolvente y persistente en el tiempo. En relación con sus dimensiones, el vigor se refiere al alto nivel de energía y resistencia mental al trabajar; la dedicación involucra una alta implicación laboral, sensación de significancia, entusiasmo, inspiración, orgullo y desafío; finalmente, la absorción representa el momento de concentración plena en la realización del trabajo, da la sensación de que el tiempo pasa rápidamente y se experimentan dificultades para desprenderse del trabajo (Salanova, Martínez, Bresó, Llorens, \& Grau, 2005).

En el ámbito académico, el engagement se relaciona con la motivación intrínseca de los alumnos por sus estudios (Bravo, 2013), ya que, desde la psicología, estudiar equivale a trabajar, puesto que los estudiantes realizan actividades estructuradas y ordenadas por alguien más con un objetivo concreto. Existen diversos estudios que muestran la eficacia del constructo en el ámbito personal y social, en el contexto académico (Schaufeli, Martínez, Marques-Pinto, Salanova, \& Bakker, 2002), por ejemplo, los estudiantes universitarios con mayores niveles de engagement se perciben auto-eficaces, tienen un nivel alto de felicidad, encuentran satisfacción en sus estudios y son menos propensos a abandonarlos (Salanova et al., 2005).

El modelo Recursos-Experiencia-Demandas [Resources-Experience-Demands model, RED] (Demerouti, Bakker, Nachreiner, \& Schaufeli, 2001), explica el proceso del engagement; este modelo, incluye cuatro variables: 1) demandasrecursos laborales, 2) recursos personales, 3) emociones-experiencias y 4) conductas laborales (Tripiana, \& Llorens, 2015). Las demandas laborales son los elementos organizacionales que exigen un coste físico y/o psicológico (Xanthopoulou, Bakker, Demerouti, \& Schaufeli, 2007). Por otro lado, los recursos laborales son los elementos sociales, físicos, psicológicos $\mathrm{y} / \mathrm{u}$ organizacionales que ayudan a alcanzar los objetivos laborales, reducir las demandas laborales y propician el crecimiento personal (Xanthopoulou et al., 2007). En relación con los recursos personales, son aspectos que pertenecen a las personas, los motivan, fomentan el aprendizaje y favorecen el desarrollo individual y colectivo, lo cual les permite enfrentar las demandas laborales (Salanova et al., 2012).

Retomado este modelo, en un estudio longitudinal, realizado en Jaume en España (Llorens, Schaufeli, Bakker, \& Salanova, 2007), se encontró que los recursos laborales (para la tarea) son factores que aumentan las creencias 
de eficacia y, a su vez, incrementan los niveles de engagement y éste de igual forma eleva la autoeficacia y genera así una espiral positiva.

En México, se han realizado pocos estudios en relación con el engagement, debido a la relativa novedad del concepto, a continuación se presentan algunos de los estudios realizados:

1. En la Universidad Nacional Autónoma de México (UNAM), Villavicencio, Jurado y Aguilar (2014a), realizaron la adaptación de la escala UWES, para medir los niveles de engagement en trabajadores; participaron 120 empleados, con un promedio de edad de 31 años y la mayoría con estudios de licenciatura. Los resultados mostraron que el UWES es apto para ser aplicado en la población laboral mexicana.

2. De igual forma, Villavicencio, Jurado $y$ Valencia (2014b), aplicaron la escala UWES, validada previamente, en una muestra de 1110 empleados de organizaciones públicas y privadas localizadas en la ciudad de México. En dicho estudio corroboraron la correlación negativa que existe entre burnout y engagement. Además, descubrieron que cuando el buen desempeño no es recompensado, el empleado pierde motivación. Igualmente, hallaron una relación positiva entre el engagement y la resiliencia, en el sentido de que la resiliencia favorece su manifestación.

3. Viejo y González (2013), realizaron un estudio en el hospital del niño morelense. Aplicaron el UWES (Schaufeli, \& Bakker,
2003) al personal de enfermería y los resultados obtenidos muestran un buen nivel de engagement entre el personal.

4. León, Romero y Olea (2012), aplicaron la escala UWES (Salanova et al., 2000) a 460 estudiantes de ingeniería de la Universidad de Sonora (UNISON), México, con la finalidad de validarla en esta población. Sin embargo, los resultados reportados indicaron que la escala no era válida ni confiable para la población mexicana. Probablemente a causa de las estrategias metodológicas utilizadas y la versión de la escala que usaron, ya que ésta fue diseñada para trabajadores, no para estudiantes.

5. En contraparte, se realizó un estudio en universitarios para validar la escala UWES-S, diseñada en exclusivo para medir los niveles de engagement en estudiantes (Schaufeli, \& Bakker, 2003). Para este estudio se retomó la versión española de la escala y con base en el método culturalmente relevante de Reyes-Lagunés y García y Barragán (2008), se realizó la validación en 721 estudiantes de psicología de tres universidades del país, cuyo resultado fue una escala trifactorial de quince reactivos, con un alfa de 0.88 , válida y confiable para ser aplicada en población universitaria mexicana (De la Cruz, Reséndiz, Romero, \& Domínguez, en prensa).

En cuanto a los valores, son concebidos como una construcción sociocultural abstracta (Schwartz, 1992) y definidos como "conceptos 
o creencias que pertenecen a estados o comportamientos finales deseados, trascienden situaciones específicas, guían la selección o la evaluación de la conducta y los eventos y están clasificados por importancia relativa" (Schwartz, 1992, p. 4). Dicha selección de comportamiento está permeada por emociones que otorgan a los valores una concepción de "principios en la vida", los cuales motivarán y guiarán las acciones y actitudes de las personas (Schwartz, 2010). Con base en diversos estudios empíricos, Schwartz (2010) identificó diez motivaciones básicas en los valores, compartidas por diversas culturas:

1. Poder: estatus social, prestigio y control sobre personas y recursos (riqueza, posición social de poder).

2. Logro: éxito personal (basado en normas sociales).

3. Hedonismo: satisfacción de las necesidades orgánicas y placer.

4. Estimulación: búsqueda de emoción, novedades y retos.

5.Autodirección: pensamiento independiente, elección de las conductas, capacidad de creación y exploración.

6. Universalismo: comprensión, aprecio, tolerancia y protección de las personas y la naturaleza.

7. Benevolencia: preservar y mejorar el bienestar de aquellas personas con las que existe mayor interacción (mostrarse útil, ser leal, brindar amistad y amor).
8. Tradición: aceptar, respetar $\mathrm{y}$ comprometerse con las costumbres e ideologías de la cultura o religión.

9. Conformidad: control de las conductas o tendencias que pudieran dañar a los demás $\mathrm{y}$ transgreden las expectativas y normativas sociales.

10. Seguridad: armonía y estabilidad social.

En cuanto a valores morales, en México existen dos estudios realizados al respecto: el primero, realizado por Kepowicz (2003) en una universidad de Guanajuato, identificó una tendencia a pensar que no es malo incumplir con los valores, ya que en el mundo existen engaños, injusticias, prepotencias y por ello es necesario protegerse mediante la búsqueda de intereses individuales, de seguridad y bienes. El segundo estudio, realizado en la UADY (Benois, 2011) se encontró que los alumnos de la licenciatura en educación consideran que los valores característicos de un profesional son la responsabilidad, ética, honestidad, respeto, puntualidad y justicia.

Otro elemento importante en esta investigación es la moral. Desde la psicología social, la moral hace referencia al código de conducta de un sujeto o grupo en particular (Luco, 2014). Estas pautas de comportamiento se establecen en forma de reglas al prohibir o permitir ciertas acciones, cuyo cumplimiento se mantiene por prácticas sociales que recompensan o castigan al sujeto, lo cual a su vez permite la internalización de dichas normas por medio de 
ciertos estados psicológicos como las actitudes, motivaciones, juicios, etcétera (Sekhar, \& Stich, 2006).

El precursor en el estudio de la moral, desde la psicología, es Kohlberg con su teoría cognitivo-evolutiva del desarrollo moral, que se enfoca en el razonamiento moral, por medio de la educación moral, y permite su desarrollo a través de etapas o estadios morales (Dewey, 1998). Su objetivo es brindar a las personas una capacidad madura y autónoma para realizar juicios éticos, evitando el adoctrinamiento al enseñar valores éticos específicos de forma directa (Kohlberg, 1975).

Por otro lado, Piaget (s. f. en Estrada, 2012) concibe el desarrollo moral como una forma de adaptación ante el medio ambiente. La formación de valores morales e interiorización de las normas sociales en el sujeto, están en función de las consecuencias de sus actos y el mantenimiento del orden social que, posteriormente, le brindarán los elementos para analizar y criticar dichas normas y valores.

Similar a lo anterior, Vigotsky (s. f. citado en Álvarez, 1998) plantea que el desarrollo moral es un proceso de aprendizaje social oadaptación, que inicia en la niñez con el seguimiento de normas o conductas establecidas. Posteriormente, en un nivel de desarrollo superior, se estructurará una visión moral internalizada del medio donde se vive, lo cual permitirá la autorregulación del comportamiento sin condicionamientos.

Por su parte, Bandura (2001) en su Teoría Social Cognitiva menciona que las personas no sólo responden ante el medio, sino que se autoexaminan a sí mismas por medio de la agencia moral, la cual sirve como regulador de conducta a través de un razonamiento moral que juzga lo correcto o incorrecto de las acciones y las compara con los estándares personales; evalúa las situaciones, circunstancias y auto-sanciones que, al final, se traducen en la realización o inhibición de la conducta.

Por último, la satisfacción, desde la psicología, es concebida como un fenómeno que se origina en la persona con base en su percepción e intereses (Jiménez et al., 2011). Es una estimación personal respecto a las expectativas, deseos y objetivos, los cuales se basan en criterios personales, influidos a la vez, por la cultura y la sociedad de la persona (Molero, 2003). Por lo tanto, la satisfacción implica la gratificación de las necesidades psicosociales del entorno, esto a la vez propicia una mayor dedicación a las actividades que son fuente de dicha satisfacción (Fuentes, 2012).

De tal forma, en el ambiente académico universitario la satisfacción académica se define como "el nivel del estado de ánimo que poseen los estudiantes con respecto a su institución, como resultado de la percepción que poseen con respecto al cumplimiento de sus necesidades, expectativas y requisitos" (Mejías, \& Martínez, 2009 , p.34). Es por esto que la satisfacción se refiere al bienestar del alumno en relación con sus actividades académicas, además, se traduce en una actitud positiva hacia la universidad y la carrera (Salanova et al., 2005). En dicho 
contexto, una institución educativa de calidad será aquella que se preocupe por infundir en sus alumnos un interés genuino hacia el aprendizaje, esta calidad a la vez es la confirmación, la satisfacción del alumno (Jiménez et al., 2011).

Dentro de las teorías que explican esta variable se encuentra la teoría social cognitiva de Bandura (2001) que permite acercarnos a la comprensión sobre la satisfacción académica. El autor hace mención sobre la capacidad de las personas para motivarse y guiar sus acciones partiendo de sus experiencias previas, esto englobado bajo el término de Reflexión (Bandura, 2001). En el contexto académico, las experiencias vividas en la universidad (respecto a las expectativas propias del alumno) y cómo las ven realizadas, con base en sus esfuerzos propios, permitirán la construcción de creencias de eficacia en concordancia con lo logrado académicamente, lo cual conducirá a la toma de decisiones tomando en cuenta la percepción de sus capacidades y recursos (Cabrera, \& Galán, 2003; Mejías, \& Martínez, 2009). Estas decisiones se toman por medio de la exploración, manipulación e influencia en el ambiente, permitiendo la regulación de la conducta (Bandura, 2001).

A continuación, se presentan estudios que se han realizado en ambientes universitarios, sobre satisfacción académica.

1. Fernández, Fernández, Álvarez y Martínez (2007), realizaron un estudio a 6,354 alumnos de la Universidad de Oviedo, España, y encontraron que la satisfacción se relaciona directamente con el éxito en las materias.

2. Hernández, Lara, Ortega, Martínez y Avelino (2010), realizaron una investigación a pequeña escala a 50 alumnos y centraron su estudio en la satisfacción percibida por los estudiantes. Los resultados mostraron que existe una satisfacción positiva a los factores intrínsecos (se perciben competentes en el estudio y con expectativas con la carrera) y una concepción negativa a lo extrínseco (insatisfacción con su institución).

3. Un estudio realizado en Tanzania, con una muestra de 260 estudiantes universitarios, mostró la relevancia del locus de control en el rendimiento académico, donde los alumnos con locus de control externo tienden a ser apáticos, conformistas, presentan bajo desempeño $\mathrm{y}$, por ende, baja productividad a causa de la insatisfacción que presentan (Mkumbo, \& Amani, 2012).

4. En México, se realizó una investigación en la Universidad Autónoma de Nayarit [UAN] (Jiménez et al., 2011) en una muestra de 960 estudiantes universitarios y se encontró que $58 \%$ es el promedio de satisfacción en los alumnos. En relación con tales resultados, los autores recomiendan a las instituciones realizar acciones tales como revisar y actualizar currículos y perfiles de egreso, actualizar a los y las docentes, estandarizar metodologías efectivas de enseñanza y aprendizaje, mejorar los procesos de evaluación e invertir en la infraestructura. 
Por estas razones, el objetivo de este estudio se plantea encontrar la relación que existe entre las variables, engagement, valores morales y satisfacción académica en estudiantes universitarios de la licenciatura en psicología de la UAEH, para tener una noción sobre la calidad de la formación académica, moral y personal de los futuros psicólogos.

\section{Método}

\section{Participantes}

Se realizó un diseño no experimental, transversal, correlacional. La selección de la muestra se realizó utilizando un muestreo no probabilístico. Participaron 215 estudiantes de la licenciatura en psicología del Instituto de Ciencias de la Salud, pertenecientes a la UAEH, 166 mujeres $(77 \%)$ y 49 hombres $(23 \%)$, con un promedio de edad de 20 años, con un rango de 17 a 42 años; 14\% fueron de primer semestre, $15 \%$ de segundo, $7 \%$ de tercero, $15 \%$ de cuarto, $5 \%$ de quinto, $7 \%$ de sexto, $13 \%$ de séptimo, $10 \%$ de octavo y $14 \%$ de noveno semestre.

\section{Instrumentos}

Para medir las variables se emplearon los siguientes instrumentos:

Satisfacción académica: para su medición se utilizó la Escala de satisfacción académica (Rojas, 1997), que evalúa el grado de satisfacción del estudiante por alcanzar los requisitos y exigencias académicas. Es una escala tipo Likert con opciones de respuesta que van de 1 (en el 10\% de ellas) a 4 (en el $90 \%$ de ellas). Tiene un alfa de Cronbach de 0.89, se compone por 21 afirmaciones, repartidas en dos factores: Aspectos generales y Teoría, con catorce afirmaciones, y Prácticas y laboratorios con siete afirmaciones.

Valores morales: se evaluó a partir del Cuestionario de valores morales en la formación y el ejercicio profesional (Herrera, 1995), que evalúa las reglas o normas de conducta asumidas como válidas dentro del ambiente de la formación profesional y en el ejercicio de la profesión. Es una escala tipo Likert, formada por 31 afirmaciones con opciones de respuesta que van de 1 (totalmente en desacuerdo) a 5 (totalmente de acuerdo). Tiene un alfa de Cronbach de 0.85 . Cuenta con dos dimensiones: Moralidad hacia lo individual $(\alpha=.83)$, presente en 22, afirmaciones y la Moralidad hacia lo social $(\alpha=.32)$, evaluada en nueve afirmaciones.

Engagement: para su medición se utilizó la escala validada para población mexicana, el Utrecht Work Engagement Scale [UWE-S México, versión para estudiantes] (De la Cruz et al., en prensa), que valora los tres elementos que integran el engagement, el alpha de Cronbach total de la escala es de .889. Está conformada por quince afirmaciones en escala de Likert con siete opciones de respuesta que van de 0 (nunca) a 6 (siempre). El alpha de Cronbach por factor es: Vigor $\alpha=.834$, Dedicación: $\alpha=.780$ y Absorción: $\alpha=.751$. 


\section{Procedimiento}

Se realizaron las gestiones pertinentes para realizar las aplicaciones en las instalaciones del Instituto de Ciencias de la Salud. Se localizó a los participantes en un horario de 8 am a 8 pm, arbitrariamente se les invitó a participar. La invitación inició con la lectura del consentimiento informado en el cual se exponían los objetivos del estudio, y se reiteraba la confidencialidad y el anonimato de la participación, posteriormente se leían las instrucciones de cada instrumento.

El análisis estadístico se realizó con el uso del programa SPSS 23.0. Se llenó una base de datos, de la cual se eliminaron los cuestionarios incompletos, considerando únicamente aquellos que tuvieran más de $80 \%$ de respuestas. Se utilizó la prueba estadística de correlación producto-momento de Pearson, al hacer las correlaciones de las tres variables, de dos en dos (Engagement-Satisfacción académica, Engagement-Valores morales, Satisfacción académica-Valores morales).

\section{Resultados}

Se presentan a continuación las medidas de tendencia central, de dispersión y los coeficientes de fiabilidad alpha de Cronbach obtenidas en cada variable (ver tabla 1).

Tabla 1

Medidas de tendencia central, de dispersión e índices de fiabilidad para las variables engagement, valores morales y satisfacción académica

\begin{tabular}{lcccc}
\hline & Escala & Media & D. E. & $\alpha$ \\
\hline Engagement & & & & \\
Vigor & $0-6$ & 3.88 & 1.17 & .844 \\
Dedicación & $0-6$ & 5.34 & .69 & .699 \\
Absorción & $0-6$ & 3.94 & 1.10 & .757 \\
Valores morales & & & & .785 \\
Moralidad hacia lo individual & $1-5$ & 4.34 & .41 & .077 \\
Moralidad hacia lo social & $1-5$ & 3.38 & .40 & .866 \\
Satisfacción académica & & & .54 & .681 \\
Aspectos generales y teoría & $1-4$ & 2.99 & .63 & \\
Prácticas y laboratorios & $1-4$ & 2.87 & & \\
\hline
\end{tabular}


Se muestran a continuación los resultados de la correlación entre las variables:

En relación con las variables, Engagement y Satisfacción académica, se encontraron relaciones estadísticamente significativas, bajas y directamente proporcionales entre el factor Aspectos generales y teoría de la Escala de Satisfacción Académica y los tres factores de la escala de Engagement. No existieron correlaciones estadísticamente significativas entre el factor Prácticas y laboratorios y los factores de Engagement (ver tabla 2).

Con respecto a las variables, Engagement y Valores morales, se encontró una asociación lineal estadísticamente significativa, baja y directamente proporcional entre el factor Valores hacia lo individual y dos factores de engagement: Dedicación y Absorción. También hubo asociaciones positivas, entre el factor Moralidad hacia lo social y Absorción (ver tabla 3).

Tabla 2

Correlación entre engagement y satisfacción académica

\section{Satisfacción académica}

\section{Factores}

\begin{tabular}{llcc}
\hline & & Aspectos generales y teoría & Prácticas y laboratorios \\
\hline \multirow{3}{*}{ Engagement } & Vigor & $.313^{* *}$ & .106 \\
& Dedicación & $.280^{* *}$ & .093 \\
& Absorción & $.191^{* *}$ & .122 \\
\hline
\end{tabular}

${ }^{*} p \leq .05^{* *} p \leq .01$

Tabla 3

Correlación entre Valores morales y Engagement

\begin{tabular}{clcc}
\hline Factores & \multicolumn{2}{c}{ Valores morales } \\
\hline \multirow{3}{*}{ Engagement } & & Moralidad hacia lo individual & Moralidad hacia lo social \\
\cline { 3 - 4 } & Dedicación & $.249^{\star *}$ & .058 \\
& Vigor & .125 & .121 \\
& Absorción & $.156^{\star}$ & $.173^{\star}$ \\
\hline
\end{tabular}

${ }^{*} p \leq .05^{* *} p \leq .01$

Finalmente, en la tercera correlación, significativa, baja y directamente proporcional, Valores morales y Satisfacción académica, se entre el factor de Moralidad hacia lo social y los encontró una relación lineal estadísticamente dos factores de satisfacción académica: Aspectos 
generales y teoría y Prácticas y laboratorios. En relación con moralidad hacia lo individual, los resultados indican que no existen relaciones estadísticamente significativas con Aspectos generales y teoría (ver tabla 4).

Tabla 4

Correlación entre Satisfacción académica y Valores morales

\begin{tabular}{llcc}
\hline & \multicolumn{2}{c}{ Satisfacción académica } \\
\hline & Factores & Aspectos generales y teoría & Prácticas y laboratorios \\
\hline $\begin{array}{l}\text { Valores } \\
\text { lo social }\end{array}$ & $.281^{* *}$ & $.149^{*}$ \\
& $\begin{array}{l}\text { Moralidad hacia } \\
\text { lo individual }\end{array}$ & .123 & .044 \\
${ }^{*} \mathrm{p} \leq .05^{* *} \mathrm{p} \leq .01$ & &
\end{tabular}

\section{Discusión}

Este estudio proporciona evidencia sobre la relación existente entre Engagement, Satisfacción académica y Valores morales. Aporta nuevos elementos a la literatura existente de valores desde la psicología y satisfacción académica, además integra nuevas variables que se relacionan con el engagement.

Los resultados muestran la existencia de una relación positiva entre Engagement y Satisfacción académica, es decir, que aquellas personas que perciben la aplicación teórica y práctica como más satisfactoria, también reportaron un mayor nivel de engagement.

La relación de Engagement y Valores morales se comprobó parcialmente. $\mathrm{La}$ Absorción se correlacionó positivamente con ambos factores de Valores morales; y la Dedicación, sólo se correlacionó con la Moralidad hacia lo individual. Teóricamente, no existen elementos para explicar estas relaciones, ya que la inclusión de valores en el campo de estudio del engagement es novedosa, aun así, es interesante el descubrimiento de la relación existente entre la absorción, que refiere a sentimiento de concentración y disfrute con la labor que se realiza (Salanova et al., 2005), y los valores morales en el ámbito académico, que se refieren al poder, logro y conformidad, según la clasificación de Schwartz (1992, 2010). Del mismo modo lo es la relación entre la Absorción (entusiasmo, inspiración, significancia, orgullo y desafío) (Salanova et al., 2005) y la Moralidad hacia lo individual (poder, logro y conformidad) (Schwartz, 1992, 2010). A manera de suposición, el porqué se relacionan estas variables puede deberse a que teóricamente cada uno de los elementos tiene relación con el buen desempeño y el éxito profesional. 
En cuanto a la relación entre Satisfacción académica y Valores morales, sólo el factor de Moralidad hacia lo social se correlacionó positivamente con ambos factores de Satisfacción académica (Aspectos generales y teoría, Prácticas y laboratorios). En teoría la relación podría explicarse de la siguiente manera: de acuerdo a Schvarstein (2006), las instituciones, incluidas las académicas, están compuestas por valores, creencias, etc. y su función principal es formar a los estudiantes para que puedan desempeñarse adecuadamente en el campo laboral, siendo muestra de su calidad, la satisfacción académica del alumno. Esta variable se correlacionó con el factor de valores morales: moralidad hacia lo social, compuesto por reactivos que hacen referencia al logro y el poder, dos de las motivaciones básicas de los valores propuestas por Schwartz (1992, 2010). Ambas variables se refieren a elementos relacionados con la preparación y el logro profesional.

No obstante, es importante tener en cuenta que el índice de consistencia interna del factor Moralidad hacia lo social se encuentra por debajo de los criterios psicométricos aceptables, por ende los resultados de la correlación con ambos factores de Satisfacción académica y el factor de Absorción pueden no ser confiables.

En referencia a los niveles de engagement, los estudiantes reportan puntajes por encima de la media teórica en los tres factores de la escala, además, en comparación con dos estudios realizados en universitarios españoles (Salanova et al., 2005; Pacheco, Durán, \& Rey, 2007), los alumnos mexicanos de esta muestra poseen un mayor nivel de engagement.

Igualmente, los puntajes de las escalas de Satisfacción académica y Valores morales se encuentran arriba de la media teórica. Estos resultados implican que altos niveles de engagement se relacionan con niveles altos de valores morales y con niveles moderados de satisfacción académica. En conclusión, el aporte más importante de esta investigación fue hallar la relación entre las variables de Engagement, Valores morales y Satisfacción académica. Aunque las correlaciones fueron bajas, son estadísticamente significativas, es decir, existe una influencia mutua entre las variables, que no se debe al azar.

\section{Alcances y limitaciones}

Dentro de los alcances más importantes de este estudio se encuentra la contribución al marco teórico del engagement académico, ya que al ser un constructo que surge en la psicología positiva organizacional, su estudio en ambientes académicos ha sido poco explorado, a pesar de su importancia, ya que investigaciones anteriores en otros países, refieren que altos niveles de engagement disminuyen la deserción (Archambault, Janosz, Fallu, \& Pagani, 2009) y mejoran la satisfacción académica (Caballero, Abello, \& Palacio, 2007). En lo que respecta al presente estudio, se muestra evidencia de la relación que existe entre esta variable, con los Valores morales y la Satisfacción académica. 
Por otra parte, en relación con los valores morales, existen muchas recomendaciones que remarcan la importancia de su estudio en ambientes académicos, por ejemplo, se menciona que las universidades se enfocan más en la preparación teórica y técnica que en la moral (Barba, 2007), aunado a la afirmación que considera los problemas por los cuales atraviesa la sociedad actualmente, en el ámbito económico, político, social, etc., son causados, en gran medida, por personas educadas (Arana, 2006). Sin embargo, a pesar de estas y otras referencias en cuanto al estudio de valores, es un tema poco explorado desde la psicología. Por lo tanto, un aporte importante de este estudio fue poder medir dicha variable; se encontró que los alumnos de esta muestra refieren valores morales superiores a la media teórica, principalmente en la Moralidad hacia lo individual, refiriendo también que esta variable se relaciona con el Engagement y la Satisfacción académica.

En cuanto a la satisfacción académica, este estudio permite visualizar que los estudiantes cuyos niveles de engagement y valores morales están por encima de la media teórica, también reportan niveles aceptables de satisfacción, lo cual coincide con lo planteado en la teoría, ya que la satisfacción implica la gratificación de las necesidades psicosociales del entorno, lo que genera mayor dedicación a las actividades que son fuentes de esta satisfacción (Fuentes, 2012).

En conclusión, el estudio de estas tres variables en conjunto permite visualizar, en menor medida, la educación superior en
México, en cuanto al bienestar, satisfacción y valores del estudiante, además de evidenciar que, aunque los resultados muestran puntajes superiores a la media teórica en las escalas, es necesario continuar explorando estas variables, pues a pesar de encontrar evidencia de que existe correlación entre ellas, sería interesante explorar las causas que subyacen a dicha relación.

Entre las limitaciones de esta investigación se encuentra la obtención de datos por medio de medidas de auto-informe, lo cual pudo sesgar los datos, puesto que no se tiene la certeza de la veracidad en las respuestas de los participantes. Otra limitación es uno de los criterios de exclusión en la recolección de datos: la participación voluntaria, dado que durante la aplicación de instrumentos una gran parte de la población se negó a participar. Esto pudo haber provocado cierto sesgo en los datos obtenidos, ya que las personas participantes pudieron favorecer los resultados positivamente, debido a la naturaleza de las variables utilizadas que se refieren a personas satisfechas con su institución académica, con valores positivos hacia su carrera y con actitudes positivas hacia sus estudios.

Otra limitación de este estudio fue la baja consistencia interna de uno de los factores de la escala de Valores morales: Moralidad hacia lo social. Para futuros estudios se recomienda utilizar un instrumento con mejores propiedades psicométricas. Asimismo, se recomienda utilizar distinto método de muestreo, para asegurar una mayor representatividad de la población, al mejorar la representación por sexo, pues en este 
estudio existió mayor participación de mujeres; de igual forma, se recomienda realizar el estudio en otras zonas del país para corroborar la relación entre las variables y, por último, complementar la investigación con metodología cualitativa por medio de grupos focales o grupos $\mathrm{T}$, esto con el fin de compensar las medidas de auto-informe.

\section{Referencias}

Aguila, V. (2005). El concepto calidad en la educación universitaria: Clave para el logro de la competitividad institucional. Revista Iberoamericana de Educación, 35(5), 1-8.

Álvarez, I. (1998). La formación de valores como propósito educativo: reflexiones teóricas para la instrumentación de programas de orientación sociomoral en la escuela. Revista Pedagogía Universitaria, 3(3), 1-10.

Álvarez, J., Chaparro, E. M., \& Reyes, D. E. (2014). Servicios Educativos brindados por Instituciones de Educación Superior del Valle de Toluca. Revista Iberoamericana sobre Calidad, Eficacia y Cambio en Educación, 13(2), 5-26.

Arana, M. H. (2006). Los valores en la formación profesional. Tabula Rasa, (4), 323-336.

Archambault, I., Janosz, M., Fallu, J., \& Pagani, L. S. (2009). Student engagement and its relationship with early high school dropout. Journal of Adolescence, 32, 651-670.

Bandura, A. (2001). Social cognitive theory: an agentic perspective. Annual Review of Psychology, 52(1), 1-26.
Barba, B. (2007). Valores, formación moral y eficacia escolar. Una revisión de la investigación educativa en México. Revista Iberoamericana sobre Calidad, Eficacia y Cambio en Educación, 5(5), 32-36.

Benois, N. A. (2011). Formación de actitudes $y$ valores en ética profesional en futuros licenciados en educación. (Tesis de Maestría). México: Universidad Autónoma de Yucatán.

Bravo, L. (2013). Predictores de engagement académico en estudiantes de odontología. Revista de Educación en Ciencias de la Salud, 10(2), 86-95.

Caballero, C., Abello, R., \& Palacio, J. (2007). Relación del burnout $y$ el rendimiento académico con la satisfacción frente a los estudios en estudiantes universitarios. Avances en Psicología Latinoamericana, 25(2), 98-111.

Cabrera, P., \& Galán, E. (2003). Satisfacción escolar y rendimiento académico. Revista de Psicodidáctica, (14), 87-98.

Cambridge University Press. (2017). Cambridge English-Spanish Dictionary. Recuperado de http://dictionary.cambridge.org/dictionary/ english-spanish/engagement

Casuso, M. (2011). Estudio del estrés, engagement y rendimiento académico en estudiantes universitarios de ciencias de la salud. (Tesis Doctoral). España: Universidad de Málaga.

De la Cruz, M. A., Reséndiz, J., Romero, A., \& Domínguez, A. (en prensa). Validación de 
la escala UWES-S México, en estudiantes universitarios mexicanos. Psicología Iberoamericana, 25.

Demerouti, E., Bakker, A. B., Nachreiner, F., \& Schaufeli. (2001). The job demandsresources model of burnout. Journal of Applied Psychology, 86(3), 499-512.

Dewey, J. (1998). Experience and Education. The 60th Anniversary Edition. Indiana, Estados Unidos de América: Kappa Delta Pi.

El Economista. (2016). Ranking 2016 Universidades. Recuperado de: http:// eleconomista.com.mx/especiales/ americaeconomia/2016/10/30/las-mejoresuniversidades-mexico-ranking-2016

Estrada, O. (2012). El profesor ante la formación de valores. Aspectos teóricos y prácticos. Teoría de la Educación. Educación y Cultura en la Sociedad de la Información, 13(3), 240-267.

Fernández, J. E., Fernández, S., Álvarez, A., \& Martínez, P. (2007). Éxito Académico y Satisfacción de los Estudiantes con la Enseñanza. Universitaria. Relieve, 13(2), 203-214.

Fuentes, S. M. (2012). Satisfacción laboral y su influencia en la productividad. Estudio realizado en la delegación de recursos humanos del organismo judicial en la ciudad de Quetzaltenango. (Tesis de licenciatura inédita). Quetzaltenango, Guatemala: Universidad Rafael Landívar.
Garbanzo, G. M. (2007). Factores asociados al rendimiento académico en estudiantes universitarios, una reflexión desde la calidad de la educación superior pública. Revista Educación, 31(41), 43-63.

Hernández, C. A., Lara, B., Ortega, M. P., Martínez, M. G., \& Avelino, R. (2010). Evaluación de la satisfacción académica de los estudiantes de la licenciatura en didáctica del francés. Revista de educación y desarrollo, 5, 35-46.

Herrera, D. (1995). Los valores morales en la formación personal. (Tesis de Maestría). México: Universidad Nacional Autónoma de México.

Instituto Mexicano para la Competitividad. (2016). Compara carreras primer trimestre de 2016. Recuperado de http://imco.org.mx/ comparacarreras/\#!/carrera/311.

Jiménez, A., Terríquez, B., \& Robles, F. J. (2011). Evaluación de la satisfacción académica de los estudiantes de la Universidad Autónoma de Nayarit. Revista Fuente, 3(6), 46-56.

Kepowics, B. (2003). Valores en los estudiantes universitarios. Un tema con muchas variaciones. Reencuentro, (38), 48-56.

Kohlberg, L. (1975). Moral education for a society in moral transition. Educational Leadership, 33(1), 46-54.

León, J., Romero, L., \& Olea, J. (2012). Estudio de validez factorial del síndrome de Burnout $\mathrm{y}$ engagement en estudiantes universitarios de ingeniería. Alternativas en Psicología, 16(27), 42-53. 
Lindsay, G. (2009). Ética profesional y psicología. Papeles del Psicólogo, 30(3), 184-194.

Llorens, S., Schaufeli, W. B., Bakker, A. B., \& Salanova, M. (2007). Does a positive gain spiral of resources, efficacy beliefs and engagement exist? Computers in Human Behavior, 23, 825-841.

Luco, A. (2014). The Definition of Morality: Threading the Needle. Social Theory and Practice, 40(3), 361-387.

Mejías, A., \& Martínez, D. (2009). Desarrollo de un Instrumento para Medir la Satisfacción Estudiantil en Educación Superior. Docencia Universitaria, 10(2), 29-47.

Mkumbo, K. A. K., \& Amani, J. (2012). Perceived University Students' Attributions of Their Academic Success and Failure. Asian Social Science, 8(7), 247-255

Molero, F. (2003). Reseña de Psicología social de los valores humanos. Desarrollos teóricos, metodológicos y aplicados de María Ros y Valdiney V. Gouveia. Revista de Psicología del Trabajo y de las Organizaciones, 19(2), 215-218.

ObservatorioLaboral.(2014).Encuesta Nacional de Ocupación y Empleo. Recuperado de: http://www.observatoriolaboral.gob.mx/ola/ content/common/reporteIntegral/busquedaR eportejsf;jsessionid $=5 \mathrm{c} 96517 \mathrm{db} 793641 \mathrm{fbe} 8$ 4266f49be?idCarreraParametro=5311\&idTi poRegistroParametro $=1 \&$ idEntidadParametr $\mathrm{o}=14$ \#AnclaGrafica.
Organización para la Cooperación y el Desarrollo Económicos. (2015). PISA 2015 Resultados clave. Recuperado de https://www.oecd.org/ pisa/pisa-2015-results-in-focus-ESP.pdf

Ouweneel, E., Le Blanc, P., \& Schaufeli, W. (2011). Flourishing students: A longitudinal study on positive emotions, personal resources, and study engagement. Journal of Positive Psychology, 6, 142-153.

Pacheco, N. E., Durán, M. A. D., \& Rey, L. (2007). Inteligencia emocional y su relación con los niveles de burnout, engagement y estrés en estudiantes universitarios. Revista de Educación, (342), 239-256.

Real Academia Española. (2014). Diccionario de la lengua española (23.a ed.). Recuperado de http://dle.rae.es/?w=diccionario

Reyes-Lagunes, I., \& García y Barragán, L. (2008). Procedimiento de validación psicométrica culturalmente relevante: un ejemplo. En S. Rivera-Aragón, R. DíazLoving, R. Sánchez-Aragón., \& I. ReyesLagunes (Eds.), La Psicología Social en México (pp. 625-636). Vol. XII. México: Asociación Mexicana de Psicología Social.

Rojas, G. T. (1997). La relación entre expectativas, satisfacción y rendimiento académico en los alumnos de la Facultad de Psicología. (Tesis de Maestría). México: Universidad Nacional Autónoma de México. Salanova, M., Llorens, S., Cifré, E., \& Martínez, I. M. (2012). We need a Hero! Towards a validation of the Healthy \& Resilient 
Organization (HERO) model. Group \& Organization Management, 37, 785-822.

Salanova, M., Martínez, I., Bresó, E., Llorens, S., \& Grau, G. (2005). Bienestar psicológico en estudiantes universitarios: facilitadores y obstaculizadores del desempeño académico. Anales de Psicología, 21(1), 170-180.

Salanova, M., Schaufeli, W., Llorens, S., Peiró, J. M., \& Grau, R. (2000). Desde el 'burnout' al 'engagement': ¿una nueva perspectiva? Revista de Psicología del Trabajo y las Organizaciones, 16, 117-134.

Salanova, M., Schaufeli, W., Martínez, I., \& Bresó, E. (2009). How obstacles and facilitators predict academic performance: The mediating role of study burnout and engagement. Anxiety, Stress, \& Coping, 26, $1-18$.

Schaufeli, W. B., \& Bakker, A. (2003). Utrech Work engagement scale: Preliminary manual. Países Bajos: Utrecht University.

Schaufeli, W. B., Martínez, I., Marques-Pinto, A., Salanova, M., \& Bakker, A. (2002). Burnout and engagement in university students: A cross-national study. Journal of Cross-Cultural Studies, (33), 464-481.

Schaufeli, W. B., Salanova, M., González-Roma, V., \& Bakker, A. (2002). The measurement of burnout and engagement: A two sample confirmatory factor analytic approach. Journal of Happiness Studies, (3), 71-92.

Schvarstein, L. (2006). Psicología social de las organizaciones: nuevos aportes. (2da ed.). Buenos Aires, Argentina: Paidós.
Schwartz, S. (1992). Universals in the content and structure of values: Theory and empirical tests in 20 countries. En M. Zanna (Ed.), Advances in experimental social psychology (pp. 1-65). Nueva York: Academic Press.

Schwartz, S. H. (2010). Basic values: How they motivate and inhibit prosocial behavior. Prosocial motives, emotions, and behavior: The better angels of our nature, 14, 221-241. Sekhar, C., \& Stich, S. (2006). A Framework for the Psychology of Norms. En P. Carruthers, S. Lawrence, S. Stich (Eds.), The Innate Mind: Culture and Cognition (pp. 280-301), Oxford: Oxford University Press.

Sheppard, S. (2011). The terms of 'engagement'. Journal of Philosophy of Education, 45(1), 111-123.

Tripiana, J., \& Llorens, S. (2015). Fomentando empleados engaged: el rol del líder y de la autoeficacia. Anales de Psicología, 31(2), 636-644.

Universidad de Guadalajara. (s.f.). Consejo para la acreditación de la educación superior. Recuperado de: http://www.cusur.udg.mx/es/ sites/default/files/adjuntos/copaes.pdf

Vallerand, R. J., \& Verner-Filion, J. (2013). Making People's Life Most Worth Living: On the Importance of Passion for Positive Psychology. Terapia Psicológica, 31(1), 3548.

Viejo, S., \& Gózalez, M. (2013). Presencia de estrés laboral, síndrome de burnout y engagement en personal de enfermería del 
hospital del niño morelense y su relación con determinados factores laborales. European Scientific Journal, 9(12), 112-117.

Villavicencio, E., Jurado, S., \& Aguilar, J. (2014a). Adaptación de las escalas UWES y OSI para trabajadores mexicanos. Psicología Iberoamericana, 22(2), 6-15.

Villavicencio, E., Jurado, S., \& Valencia, A. (2014b). Work engagement and occupational burnout: it's relation to organizational socialization and psychological resilience. Journal of Behavior, Health \& Social Issues, 6(2), 45-55.

Xanthopoulou, D., Bakker, A., Demerouti, E., \& Schaufeli, W. B. (2007). The Role of Personal Resources in the Job DemandsResources Model. International Journal of Stress Management, 14(2), 121-141.

Recibido el 19 de octubre de 2016 Revisado el 7 de noviembre de 2016 Aceptado el 15 de noviembre de 2016 